\title{
CARACTERIZAÇÃO FÍSICO-QUÍMICA DE QUEIJO PRATO POR ESPECTROSCOPIA NO INFRAVERMELHO E REGRESSÃO DE MÍNIMOS QUADRADOS PARCIAIS
}

\author{
Elenise Sauer-Leal e Fernanda Martins Okada \\ Departamento de Alimentos, Universidade Tecnológica Federal do Paraná, Campus Ponta Grossa, Av. Monteiro Lobato, \\ s/n - km 04, 84016-210 Ponta Grossa - PR, Brasil \\ Patricio Peralta-Zamora* \\ Departamento de Química, Universidade Federal do Paraná, CP 19081, 81531-990 Curitiba - PR, Brasil
}

Recebido em 11/6/07; aceito em 24/4/08; publicado na web em 22/9/08

\begin{abstract}
PHYSICO-CHEMICAL CHARACTERIZATION OF PRATO CHEESE BY INFRARED SPECTROSCOPY AND PARTIAL LEAST SQUARES REGRESSION. In this work an analytical methodology for the determination of relevant physicochemical parameters of prato cheese is reported, using infrared spectroscopy (DRIFT) and partial least squares regression (PLS). Several multivariate models were developed, using different spectral regions and preprocessing routines. In general, good precision and accuracy was observed for all studied parameters (fat, protein, moisture, total solids, ashes and $\mathrm{pH}$ ) with standard deviations comparable with those provided by the conventional methodologies. The implantation of this multivariate routine involves significant analytical advantages, including reduction of cost and time of analysis, minimization of human errors, and elimination of chemical residues.
\end{abstract}

Keywords: prato cheese; DRIFTS; multivariate calibration.

\section{INTRODUÇÃO}

Para priorizar o fornecimento de produtos alimentícios seguros, uniformes e com qualidade, o sistema produtivo precisa manter um rigoroso controle de qualidade, atendendo critérios de órgãos fiscalizadores e demandas de mercado. ${ }^{1}$ Em função desta necessidade, a disponibilização de métodos analíticos eficientes e rápidos torna-se prioritária, principalmente em casos em que as metodologias tradicionais se mostram demoradas, dispendiosas, inseguras e incompatíveis com as novas diretrizes ambientais. ${ }^{2}$

Dentro do contexto dos produtos lácteos, controles que visam o cumprimento de padrões relacionados com identidade e qualidade são previstos pelo Ministério da Agricultura, ${ }^{3}$ sendo as normas de rotulagem previstas pela Agência Nacional de Vigilância Sanitária. ${ }^{4}$ No caso particular do queijo prato, a caracterização físico-química envolve determinações de extrato seco, umidade, proteína, gordura, cinzas e $\mathrm{pH}$, parâmetros determinados por metodologias oficiais, reconhecidamente demoradas e geradoras de resíduos.

Nos últimos anos, metodologias que utilizam técnicas instrumentais e ferramentas de calibração multivariada têm sido abundantemente descritas na literatura, com o objetivo de reduzir o tempo de análise e o gasto de reagentes, mantendo a eficiência e a confiabilidade das metodologias convencionais.

Dentro deste contexto, a espectroscopia no infravermelho aparece como ferramenta de primeira importância, permitindo o estabelecimento de inúmeras metodologias analíticas orientadas ao controle de qualidade de produtos alimentícios.$^{5-8}$ Além de versátil, permitindo a análise de uma grande diversidade de matrizes de interesse, a técnica apresenta-se precisa, rápida e segura, ${ }^{8-10}$ o que favorece a sua utilização na implementação de rotinas de trabalho. ${ }^{11}$

Levando-se em consideração os objetivos deste trabalho, resulta especialmente interessante destacar o trabalho de Vannini, ${ }^{12}$ que demonstra a capacidade da espectroscopia no infravermelho para o mo-

*e-mail: zamora@quimica.ufpr.br nitoramento de mudanças físico-químicas na estrutura de proteínas, em consequiência da atividade proteolítica microbiana desenvolvida durante a maturação de queijos, e o trabalho de Mcqueen et al, ${ }^{13}$ que demonstra a viabilidade da caracterização química de queijos, recorrendo-se à espectroscopia no infravermelho.

Dentre os vários métodos multivariados disponíveis, destaque pode ser dado à regressão de mínimos quadrados parciais (PLS), método que se mostra mais eficiente para contornar problemas associados a ruído instrumental e colinearidade. ${ }^{14}$ Os fundamentos deste processo de calibração são bem conhecidos, podendo ser consultados na literatura recente. ${ }^{15,16}$

A análise multivariada vem sendo maciçamente utilizada na área de alimentos, permitindo a análise sensorial de leite, ${ }^{17}$ a caracterização sensorial e de textura de queijo $\operatorname{cheddar~}^{18}$ e a correlação química e sensorial de queijo parmigiano-reggiano, ${ }^{19}$ dentre outros. De acordo com Adamopoulos et al.,${ }^{20}$ a associação entre espectroscopia no infravermelho próximo (NIR) e ferramentas de calibração multivariada permite a redução de erros humanos e o aumento da freqüência de análise.

O presente trabalho apresentou como principal objetivo o desenvolvimento de uma metodologia analítica orientada ao controle de qualidade de queijo prato, utilizando espectroscopia no infravermelho por refletância difusa (DRIFT) e regressão de mínimos quadrados parciais.

\section{PARTE EXPERIMENTAL}

\section{Amostra e amostragem}

Amostras de queijo prato de diferentes marcas e procedências foram adquiridas em estabelecimentos comerciais das cidades de Ponta Grossa e Curitiba, sendo transportadas até o laboratório em temperatura controlada $\left(4-7^{\circ} \mathrm{C}\right)$. Cada amostra foi identificada numericamente e dividida em duas partes. Uma parte de 250 a $300 \mathrm{~g}$ foi reservada para análises físico-químicas convencionas, enquanto que a segunda, de 50 a 100 g, foi reservada para análise espectroscópica. 
As amostras destinadas a análises físico-químicas foram trituradas, acondicionadas em pacotes de polietileno e conservadas sob refrigeração até a execução das análises, enquanto que as amostras destinadas à análise espectroscópica foram mantidas na sua forma original, em bloco ou fatias. Todas estas análises foram realizadas na mesma semana em que as amostras foram adquiridas.

Das 25 amostras coletadas, 20 foram utilizadas para elaboração dos modelos, enquanto que as 5 restantes foram reservadas para a fase de validação.

\section{Análises físico-químicas}

As análises físico-químicas de gordura, proteína, umidade, extrato seco, cinzas e $\mathrm{pH}$ foram realizadas conforme método oficial da AOAC (Association of Official Analytical Chemists). ${ }^{21}$ Com exceção de gordura, determinada em duplicata, todas as análises foram realizadas em triplicata.

\section{Determinação de gordura}

Para determinação do teor de gordura, amostras de 2,5 g foram moídas, homogeneizadas e pesadas diretamente em butirômetro de Van Gulik, especial para queijo. Após adição de $5 \mathrm{~mL}$ de água a $42^{\circ} \mathrm{C}$ e $10 \mathrm{~mL}$ de $\mathrm{H}_{2} \mathrm{SO}_{4}\left(\mathrm{~d}=1,825 \mathrm{~g} / \mathrm{cm}^{3}\right)$ este conteúdo foi agitado moderadamente e colocado em banho-maria a $65^{\circ} \mathrm{C}$, até total dissolução da amostra. Após dissolução e adição de $1 \mathrm{~mL}$ de álcool isoamílico, os butirômetros foram completados com água aquecida a $30{ }^{\circ} \mathrm{C}$, sendo finalmente centrifugados por $5 \mathrm{~min}$. $\mathrm{O}$ volume de gordura foi medido diretamente na escala do butirômetro, expresso em porcentagem.

\section{Determinação de proteína}

O conteúdo de nitrogênio total dos queijos foi determinado empregando-se o método micro-Kjedahl, compreendendo as fases de digestão, destilação e titulação.

$\mathrm{Na}$ etapa de digestão, $0,1 \mathrm{~g}$ de queijo foi pesado em tubos micro Kjedahl e adicionado de $0,5 \mathrm{~g}$ de catalisador (sulfato cúprico e sulfato de potássio) e $5 \mathrm{~mL}$ de ácido sulfúrico concentrado. Em seguida, a amostra foi digerida por $1 \mathrm{~h}$ a temperatura de $250{ }^{\circ} \mathrm{C}$ e mais $1 \mathrm{~h}$ a $350{ }^{\circ} \mathrm{C}$, permanecendo na temperatura de $400{ }^{\circ} \mathrm{C}$ até finalização da digestão. Decorrido este tempo esfriou-se o tubo e adicionaram-se $10 \mathrm{~mL}$ de água destilada para iniciar a destilação.

Para destilação, o tubo de micro-Kjedahl com o conteúdo digerido foi adaptado ao sistema, neutralizando-se com aproximadamente 30 $\mathrm{mL}$ de $\mathrm{NaOH} 30 \%$. O destilado foi recolhido em erlenmeyer de 250 $\mathrm{mL}$, contendo $10 \mathrm{~mL}$ de ácido bórico $4 \%(\mathrm{~m} / \mathrm{v})$.

Finalmente, a titulação foi realizada com $\mathrm{H}_{2} \mathrm{SO}_{4} 0,01 \mathrm{~mol} \mathrm{~L}^{-1}$, na presença de indicador misto, composto por vermelho de metila e azul de metileno.

O volume gasto foi utilizado para cálculo de $\%$ de nitrogênio total contido nas amostras de queijo e convertido a \% de proteína utilizando-se o fator 6,38 (fator de conversão de nitrogênio para proteína de leite e produtos lácteos).

\section{Determinação de extrato seco e umidade}

O teor de extrato seco total foi obtido gravimetricamente, utilizando estufa convencional, enquanto que o teor umidade foi determinado por diferença. Para tal, $3 \mathrm{~g}$ de amostra de queijo foram colocadas em uma placa de alumínio contendo areia purificada, sistema este previamente seco e tarado. Após homogeneização, a amostra foi colocada em estufa a $104{ }^{\circ} \mathrm{C}$ por $7 \mathrm{~h}$. Em seguida as placas foram transferidas para dessecadores para resfriamento e pesagem.

\section{Determinação de cinzas}

O conteúdo de cinzas dos queijos foi determinado a partir de 2 $\mathrm{g}$ da amostra, as quais foram pesadas diretamente em cadinho de porcelana previamente tarado. Em seguida, carbonizou-se a amostra em chapa aquecedora, de modo a se evitar o espalhamento e a perda da gordura. Finalmente, a amostra foi colocada em mufla a $540{ }^{\circ} \mathrm{C}$ por $18 \mathrm{~h}$. Ao final desse período os cadinhos foram transferidos para dessecadores para resfriamento e pesagem.

\section{Determinação do $\mathrm{pH}$}

Em um béquer de $50 \mathrm{~mL}$ pesaram-se $20 \mathrm{~g}$ da amostra triturada de queijo, adicionando em seguida $20 \mathrm{~mL}$ de água destilada. Após homogeneização o $\mathrm{pH}$ foi medido diretamente, utilizando-se potenciômetro digital TECNAL, modelo TEC-2MP.

\section{Análises espectroscópicas}

As análises espectroscópicas foram realizadas em espectrofotômetro infravermelho da série Excalibur BIORAD, FTS 3500GX, equipado com beamsplitter de $\mathrm{KBr}$; detector de sulfeto de triglicerina deuterada (DTGS), fonte de radiação de carbeto de silício e acessório de refletância difusa (DRIFT). As medidas foram realizadas entre 7500 e 400 $\mathrm{cm}^{-1}$, com resolução de $4 \mathrm{~cm}^{-1}$ e acumulação de 128 varreduras.

As amostras foram apresentadas na forma de fatias, de $10 \mathrm{~mm}$ de diâmetro e $3 \mathrm{~mm}$ de espessura.

\section{Análises quimiométricas}

Os modelos multivariados de calibração foram desenvolvidos por uma rotina de regressão de mínimos quadrados parciais (PLSR), aplicada com software PLS-toolbox 1.5 (Eigenvector Research Inc.), operando em ambiente Matlab versão 6.5 (Mathworks Inc.). ${ }^{22}$ Para análise estatística e montagem de matrizes de dados utilizou-se o software Origin 6.1®.

Os pré-processamentos utilizados foram: alisamento pelo método Savitzky-Golay (empregando-se intervalos de alisamento de 3 a 33 pontos, representados como S3 a S33, e ajuste por polinômios de segunda ordem) seguidos de cálculo da primeira derivada e correção do espalhamento multiplicativo (MSC). Em todos os casos os espectros foram centrados na média.

Para detecção de amostras anômalas utilizaram-se os critérios de leverage e resíduos de Student. A leverage é uma medida da influência de uma amostra no modelo de regressão, apresentando um valor limite equivalente a $3 \mathrm{VL} / \mathrm{n}$ (onde VL representa o número de variáveis latentes e n o número de amostras). Supondo-se que os resíduos de Student são normalmente distribuídos, a aplicação de um teste $t$ pode verificar se a amostra está ou não dentro da distribuição com um nível de confiança de $95 \%$. Como os resíduos de Student são definidos em unidades de desvio padrão do valor médio, os valores além de $\pm 2,5$ são considerados altos sob as condições usuais da estatística. ${ }^{14}$

A exatidão dos modelos foi verificada calculando-se o grau de concordância entre o valor de referência $\left(\mathrm{y}_{\mathrm{i}}\right)$ e o valor estimado $\left(\hat{\mathrm{y}}_{\mathrm{i}}\right)$, utilizando-se os parâmetros de erro quadrático médio de calibração (RMSEC - Root Mean Squares Error of Calibration) e de previsão (RMSEP - Root Mean Squares Error of Predicton), onde n representa o número de amostras na fase de calibração e de validação, respectivamente..$^{22,23}$

Embora exista uma equação conceitualmente mais apropriada para esta avaliação, o parâmetro foi calculado a partir da expressão apresentada abaixo, que corresponde à versão aproximada considerada pelo programa computacional utilizado.

RMSEC; RMSEP $=\left\{\sum_{i=1}^{N}\left(y_{i}-\hat{y}_{i}\right)^{2} / n\right\}^{-1 / 2}$ 


\section{RESULTADOS E DISCUSSÃO}

\section{Caracterização físico-química de queijo prato}

$\mathrm{Na}$ avaliação das características físico-químicas dos macroconstituintes (Tabela 1) foi observado, de maneira geral, que todos os valores se aproximam da composição média atribuída ao queijo prato. Isto é, umidade entre 42 e $44 \%$, proteína entre 23 e $25 \%$ e gordura entre 26 e $29 \% .^{24,25}$

Tabela 1. Características físico-químicas das amostras de queijo prato

\begin{tabular}{lccc}
\hline Parâmetro & \multicolumn{3}{c}{ Valores médios } \\
& $(\%)$ & $\mathrm{dp}$ & $\mathrm{CV}(\%)$ \\
\hline Extrato seco & 57,65 & $\pm 0,47$ & 0,82 \\
Umidade & 42,36 & $\pm 0,47$ & 1,11 \\
Proteína & 23,65 & $\pm 0,36$ & 1,52 \\
Gordura & 28,68 & $\pm 0,43$ & 1,50 \\
Cinzas & 4,04 & $\pm 0,03$ & 0,74 \\
pH & 5,50 & $\pm 0,01$ & 0,18 \\
\hline
\end{tabular}

Média (\%): Média dos parâmetros em \%, dp: estimativa do desvio padrão, CV: coeficiente de variação.

Estes resultados corroboram a afirmação de Sanches ${ }^{26}$ que relata o queijo prato como bem padronizado tecnologicamente e o melhor caracterizado comercialmente, complementando ainda que a aceitabilidade do consumidor a este produto está relacionada com as suas características físico-químicas e sensoriais.

Uma maior variabilidade foi observada nos valores de cinzas (3,60 a 4,37\%) e pH (4,89 a 6,25). Este último, inclusive, apresenta variabilidade maior que o intervalo citado por Furtado, ${ }^{24} \mathrm{com}$ valores entre 5,2 e 5,4 .

Tanto os valores dos macro-constituintes quanto os valores de cinzas e pH indicam diferenças significativas entre as amostras, mesmo se tratando do mesmo tipo de produto. Entretanto, trata-se de valores que se distribuem de maneira homogênea, o que viabiliza o desenvolvimento de modelos de calibração.

Finalmente, cabe salientar que, embora constituídas de diversas etapas, algumas demoradas e de difícil execução (ex. determinação de proteínas), as rotinas de análise apresentam uma excelente repetibilidade, representada por estimativas de desvio padrão entre 0,82 $\%$ (extrato seco) e $1,52 \%$ (proteínas).

\section{Caracterização espectroscópica de queijo prato}

Um espectro infravermelho típico de amostras de queijo prato é apresentado na Figura 1. Em primeiro lugar, é interessante salientar a extrema semelhança com espectros de queijo Cheddar, apresentados por Chen et al. ${ }^{27}$

Na região média (MID), observam-se bandas de absorção entre 3760 e $1520 \mathrm{~cm}^{-1}$, principalmente relacionadas com presença de água (vibração de grupos hidroxila por deformação axial simétrica e assimétrica entre 3600 e $3000 \mathrm{~cm}^{-1}$ ), gordura (vibrações de estiramentos de grupos C-H, associadas aos grupos metil e metileno em 2930 e 2850 e sinal característico de estiramentos de grupos carbonila entre 1730 e $1765 \mathrm{~cm}^{-1}$ ), e proteínas (vibrações de estiramentos de grupos $\mathrm{N}-\mathrm{H}$ em 3240,1650 e $\left.1540 \mathrm{~cm}^{-1}\right)$. $^{28-31}$

Na região próxima (NIR) observam-se bandas de absorção entre 7500 a $4000 \mathrm{~cm}^{-1}$, associadas a sobretons e combinação de ligações de grupos C-H, N-H e O-H. ${ }^{32-35}$

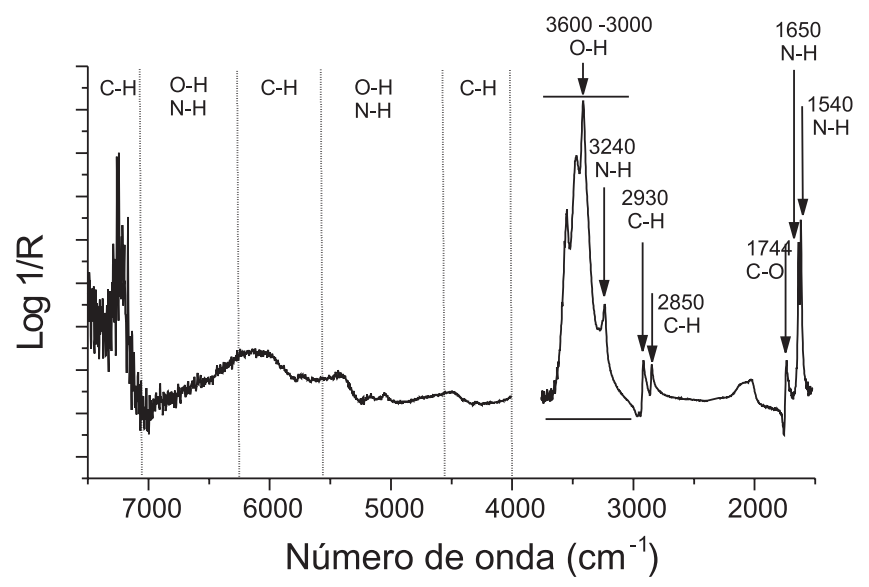

Figura 1. Espectro característico de queijo prato no infravermelho próximo de 7500 a $4000 \mathrm{~cm}^{-1}$ e infravermelho médio de 3760 a $1520 \mathrm{~cm}^{-1}$

\section{Modelos multivariados para determinação individual de parâmetros físico-químicos}

Para determinação individual de cada uma das propriedades físico-químicas avaliadas, inúmeros modelos preliminares foram desenvolvidos, principalmente objetivando selecionar a melhor região espectral, o melhor sistema de pré-processamento de sinais e o melhor número de variáveis latentes. De forma resumida, estes resultados são apresentados nas Figuras $1 \mathrm{~S}$ e $2 \mathrm{~S}$ (material suplementar), nas quais se relaciona o RMSEP com a região espectral e o número de variáveis latentes (figura da esquerda) e com a região espectral e o sistema de pré-procesamento (figura da direita).

De maneira geral, foi observado que o menor valor de RMSEP é conseguido com um grande número de variáveis latentes (VLs), normalmente 10 a 15 . Entretanto, foi também verificado que a variância representada a partir da oitava VLé pouco significativa, assim como os ganhos em capacidade de previsão. Por este motivo, e visando evitar a criação de modelos superajustados, que normalmente apresentam pouca robustez, foi decidido desenvolver modelos utilizando-se 2 a 8 VLs.

$\mathrm{Na}$ fase de calibração, excelentes valores de coeficientes de regressão foram observados (Tabela 2), assim como ausência de anomalias no conjunto de amostras utilizadas no desenvolvimento dos modelos.

A capacidade preditiva dos modelos foi avaliada por validação externa, utilizando-se 5 amostras que não fizeram parte do processo de modelagem. Para determinação do teor de gorduras e proteínas os melhores modelos de calibração foram conseguidos na região do infravermelho próximo (6800 a $4000 \mathrm{~cm}^{-1}$ ), utilizando préprocessamento centrado na média. Utilizando-se 4 VLs o modelo demonstrou boa capacidade preditiva, permitindo erros de previsão inferiores a $2 \%$ (Tabela 2).

A região espectral compreendida entre 7000 e $4000 \mathrm{~cm}^{-1}$ permitiu a elaboração de modelos com boa capacidade de previsão de umidade, extrato-seco e cinzas, utilizando-se, em todos os casos, 4 VLs. O pré-procesamento por MSC permitiu a melhor previsão de umidade e extrato seco, com erros relativos de 1,44 e 1,02\%, respectivamente (Tabela 2). Para avaliação do teor de cinzas o pré-processamento, constituído de alisamento e derivação, apresentou-se mais conveniente, permitindo erros de previsão da ordem de $2,6 \%$.

Trabalhando-se na região do infravermelho médio (3760 a 1520 $\mathrm{cm}^{-1}$ ), excluindo-se as regiões que caracterizam a absorção por parte do $\mathrm{CO}_{2}$ e as de elevado ruído instrumental, praticamente todos os modelos conseguiram reproduzir a concentração dos padrões de calibração com boa aproximação. Entretanto, destaque pode ser dado ao modelo que visa a determinação de $\mathrm{pH}$, elaborado com 6 VLs e 
Tabela 2. Resultados dos modelos de calibração PLS individuais para determinação dos parâmetros físico-químicos no infravermelho. Préprocessamentos: CM (centrado na média), MSC (correção do espalhamento multiplicativo), S5 (5 pontos de alisamento, ajuste polinomial de ordem 2 e primeira derivada) e S21 (21 pontos de alisamento, ajuste polinomial de ordem 2 e primeira derivada)

\begin{tabular}{|c|c|c|c|c|c|c|c|c|c|c|c|c|}
\hline \multirow{2}{*}{ Parâmetros } & \multicolumn{2}{|c|}{ Gordura } & \multicolumn{2}{|c|}{ Proteína } & \multicolumn{2}{|c|}{ Umidade } & \multicolumn{2}{|c|}{ Extrato seco } & \multicolumn{2}{|c|}{ Cinzas } & \multicolumn{2}{|c|}{$\mathrm{pH}$} \\
\hline & \multicolumn{12}{|c|}{ Modelagem } \\
\hline Região espectral $\left(\mathrm{cm}^{-1}\right)$ & \multicolumn{2}{|c|}{$4000-6800$} & \multicolumn{2}{|c|}{$4000-6800$} & \multicolumn{2}{|c|}{$4000-7500$} & \multicolumn{2}{|c|}{$4000-7500$} & \multicolumn{2}{|c|}{$4000-7500$} & \multicolumn{2}{|c|}{$1520-3760$} \\
\hline Pré-processamento & \multicolumn{2}{|c|}{$\mathrm{CM}$} & \multicolumn{2}{|c|}{$\mathrm{CM}$} & \multicolumn{2}{|c|}{ MSC } & \multicolumn{2}{|c|}{ MSC } & \multicolumn{2}{|c|}{ S21 } & \multicolumn{2}{|c|}{ S5 } \\
\hline VLs & \multicolumn{2}{|c|}{4} & \multicolumn{2}{|c|}{4} & \multicolumn{2}{|c|}{4} & \multicolumn{2}{|c|}{4} & \multicolumn{2}{|c|}{4} & \multicolumn{2}{|c|}{6} \\
\hline Variância explicada (\%X) & \multicolumn{2}{|c|}{99,89} & \multicolumn{2}{|c|}{99,90} & \multicolumn{2}{|c|}{99,73} & \multicolumn{2}{|c|}{99,73} & \multicolumn{2}{|c|}{88,86} & \multicolumn{2}{|c|}{98,29} \\
\hline \multirow[t]{2}{*}{ Variância explicada (\%Y) } & & & 97 & & 99 & & & & 88 & & & \\
\hline & & & & & & Calil & ação & & & & & \\
\hline RMSEC & & & 0 , & & & & & & 0 , & & & \\
\hline Rcal & & & 0 , & & & & & & 0 , & & & \\
\hline & & & & & & Pre & & & & & & \\
\hline & EXP & PRE & EXP & PRE & EXP & PRE & EXP & PRE & EXP & PRE & EXP & PRE \\
\hline Amostra 1 & 28,1 & 27,0 & 22,5 & 23,8 & 44,0 & 44,1 & 56,1 & 55,9 & 3,83 & 3,96 & 5,68 & 5,46 \\
\hline Amostra 2 & 28,2 & 29,1 & 26,5 & 25,4 & 40,5 & 41,1 & 59,5 & 58,9 & 4,01 & 3,81 & 5,54 & 5,54 \\
\hline Amostra 3 & 28,2 & 28,9 & 23,6 & 24,1 & 43,3 & 42,5 & 56,7 & 57,6 & 4,11 & 3,96 & 5,4 & 5,44 \\
\hline Amostra 4 & 29,3 & 29,7 & 24,0 & 25,6 & 41,2 & 42,0 & 58,8 & 58,0 & 3,94 & 3,93 & 5,36 & 5,27 \\
\hline Amostra 5 & 28,7 & 28,7 & 24,4 & 24,4 & 41,2 & 41,7 & 58,8 & 58,3 & 4,21 & 4,23 & 5,29 & 5,27 \\
\hline RMSEP & & & 0 , & & & & & & 0 , & & & \\
\hline Erro médio (\%) & & & 1 , & & & & & & 2 , & & & \\
\hline
\end{tabular}

pré-processamento por alisamento e derivação, que permitiu erros de previsão da ordem de 1,4\% (Tabela 2 ).

Realizando-se uma análise comparativa com resultados publicados na literatura recente, é possível observar que os modelos aqui desenvolvidos para a determinação de gordura, proteína e umidade permitiram a obtenção de erros de previsão inferiores aos descritos na análise de queijos por FTIR-ATR. ${ }^{13}$ Adicionalmente, os modelos obtidos para gordura, proteína e extrato seco apresentaram o mesmo número de VL e erros padrão de validação inferiores aos obtidos por Jankovska e Sustová, ${ }^{36}$ que utilizaram espectroscopia NIR no modo refletância para análise de leite.

Da mesma forma, o modelo orientado à determinação de cinzas apresentou um melhor desempenho que os modelos reportados por Ferrarini et al.,${ }^{37}$ que objetivam a avaliação de parâmetros nutricionais de milho por DRIFT.

Os resultados do modelo para proteína foram similares aos obtidos por Morgano et al. ${ }^{38}$ utilizando espectros NIR por refletância difusa em café.

A realização da fase experimental evidenciou a complexidade das análises por via úmida, principalmente em relação à determinação de proteínas e gorduras. Estes procedimentos se mostram onerosos, demorados e geradores de grandes quantidades de resíduos, sendo freqüentemente associados a problemas de repetibilidade, em razão do elevado número de operações envolvidas.

De maneira geral, foi possível observar que modelos MID e NIR apresentam um bom potencial de aplicação para a determinação individual dos parâmetros de interesse. Entretanto, a região do infravermelho próximo forneceu modelos de melhor desempenho, proporcionando condições para uma análise rápida e confiável.

Com relação a aspectos mais práticos, relacionados à implementação de rotinas de controle, é importante destacar o significativo aumento na oferta de sistemas instrumentais compactos, orientados à aquisição de espectros na região do infravermelho próximo. A disponibilização deste tipo de instrumental, muitas vezes aparelhado com recursos de calibração multivariada, favorece o estabelecimento de rotinas fundamentadas neste tipo de proposta.

\section{CONCLUSÕES}

Os resultados obtidos demonstram a capacidade das ferramentas de calibração multivariada no desenvolvimento de metodologias espectroscópicas (DRIFT) orientadas à determinação da composição físico-química de queijos.

Modelos multivariados individuais permitem a determinação de todos os parâmetros de interesse com exatidão e precisão, utilizandose informações espectrais do infravermelho próximo (gordura, proteína, umidade, extrato seco e cinzas) e do infravermelho médio ( $\mathrm{pH}$ ).

Os modelos multivariados desenvolvidos permitiram a obtenção de resultados comparáveis aos obtidos por aplicação da rotina convencional por via úmida.

A utilização de uma técnica instrumental sem preparo de amostras implica em inúmeras vantagens do sistema proposto, principalmente relacionadas à redução de tempo, custos, inexistência de resíduos químicos e potencial aplicação em sistemas on line.

\section{MATERIAL SUPLEMENTAR}

Disponível gratuitamente em http://quimicanova.sbq.org.br. Nas Figuras $1 \mathrm{~S}$ e $2 \mathrm{~S}$ se apresenta, de forma resumida, o efeito da região espectral processada, do tipo de pré-processamento de sinal utilizado e do número de variáveis latentes na capacidade de previsão dos modelos desenvolvidos.

\section{REFERÊNCIAS}

1. http://www.conferencia.uncnet.br, acessada em Maio 2007.

2. Geladi, P.; Spectrochim. Act, Part B 2003, 58, 767.

3. Brasil. Ministério da Agricultura e do Abastecimento; Regulamento Técnico para fixação de identidade e qualidade do queijo prato, Portaria

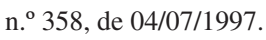

4. ANVISA. Agencia Nacional de Vigilância Sanitária; Regulamento Técnico sobre Rotulagem Nutricional de Alimentos Embalados, Resolução RDC nº. 360, 2003. 
5. Dubois, J.; Van De Voort, F.R.; Sedman, I.; Ismail, A. A.; Ramaswamy, H. R.; J. Am. Oil Chem. Soc. 1996, 73, 787.

6. Demiate, I. M.; Dupuy, N.; Huvenne, J. P.; Cereda, M. P.; Wosiacki. G.; Carbohydr. Polym. 2000, 42, 149.

7. Kavanagh, G. M.; Clark, A. H.; Ross-Murphy, S. B.; Int. J. Biol. Macromol. 2000, 28, 41.

8. Moreno, I.; Van dender, A. G. F.; Costa, G. A. N.; Vialta, A.; Lerayer, A. L. S.; Silva, A. T.; Destro, M. T.; Anais do XXI Congresso Nacional de Laticínios, Juiz de fora, Brasil, 2001.

9. Wojciechowski, C.; Dupuy, N.; Huvenne, C. D.; Ta, J. P.; Legrand, P.; FOOD CHEM. 1998, 63, 133.

10. Sansal, U.; Somer, G.; Food Chem. 1999, 65, 259.

11. Adhikari C.; Balasubramaniam, V. M.; Abbott, U. R.; Lebensm. Wiss. Technol. 2003, 36, 21.

12. Vannini, L.; Baldi, D.; Lanciotti, R.; Int. J. Food Microbiol. 2001, 69, 113.

13. Mcqueen, D. H.; Wilson R.; Kinnunen, A.; Jensen, E. P.; Talanta 1995, 42, 2007.

14. Ferreira, M. M. C.; Antunes, A. M.; Melgo, M. S.; Volpe, P. L. O.; Quim. Nova 1999, 22, 724.

15. Vigneau, E.; Qannari, E. M.; Bertra, D.; Chemom. Intell. Lab. Syst. 2002, $63,7$.

16. Feudale, R. N.; Woody, N. A.; Tan, H.; Myles, A. J.; Brown, S. D.; Ferré, J.; Chemom. Intell Lab. Syst. 2002, 64, 181.

17. Frøst, M. B.; Dijksterhuis, G.; Martens, M.; Food Qual. Pref. 2001, 12, 327.

18. Truong, V. D., Daubert, C. R., Drake, M. A.; Baxter, S. R.; Lebensm. Wiss. Technol. 2002, 35, 305.

19. Virgili, R.; Parolari, G.; Bolzoni, L.; Barbieri, G.; Mangia, A.; Careri, M.; Spagnoli, S.; Panari, G.; Zannoni, M.; Lebensm. Wiss. Technol. 1994, 27, 491.

20. Adamopoulos, K. G.; Goula, A.; Petropakis, H. J.; J. Food Comp. Anal. 2001, 14, 431.

21. AOAC. Association of Official Analytical Chemists; Official methods of Analysis, Washington. $15^{\text {th }}$ ed., 1995.
22. MatLab - The Language of Technical Computing, version 6.5; The MathWorks Inc. 2001.

23. Morgano, M. A.; Faria, C. G.; Ferrão, M. F.; Ferreira, M. M. C.; Quim. Nova 2007, 30, 346.

24. Furtado M. M., Lourenço Neto, J. P. M.; Tecnologia de queijos: manual técnico para a produção industrial de queijos, Dipemar Ltda: São Paulo, 1994.

25. Valle, J. L. E.; Moreno, I.; Dender, A. G. F. V.; Souza, G.; Cienc. Tecnol. Aliment. 1992, 22, 83.

26. Sanches, V. A. A. G.; Dissertação de Mestrado, Universidade de São Paulo, Brasil, 2000.

27. Chen, M.; Irudayaraj, J.; Mcmahon, D. J.; J. Dairy Sci. 1998, 81, 2791.

28. Silverstein, R. M.; Bassler, G. C.; Morrill, T. C.; Identificação espectrométrica de compostos orgânicos, 5a ed., Guanabara Koogan: Rio de Janeiro, 1994.

29. Surewicz, W. K.; Mantsh, H. H.; Biochim. Biophys. Acta, Proteins and Proteomics 1988, 952,115.

30. Sarver, R. W. Jr.; Krueger, W. C.; Anal. Biochem. 1991, 194, 89.

31. Belton, P. S.; Saffa, A. M.; Wilson, R. H.; Food Chem. 1988, $28,53$.

32. Murray, I. Em Sward Herbage Measurement Handbook; Davies, A.; Baker, R. D.; Grant, S. A.; Laidlaw, A. S., eds.; British Grassland Society, Reading: UK, 1993, cap. 14.

33. Batten, G. D.; Aust. J. Exp. Agr. 1998, 38, 697.

34. Deaville, E. R.; Flinn, P. C. Em Forage Evaluation in Ruminant Nutrition, Givens, D. I.; Owen, E.; Axford, R. F. E.; Omed, H. M., eds.; CABI Publishing: UK, 2000.

35. Reeves III, J.B. Em Farm Animal Metabolism and Nutrition; D’Mello, J. P. F., ed.; CABI Publishing: UK, 2000, cap. 9.

36. Jankovská, R.; Šustová, K.; Czech J. Food Sci. 2003, 21,123.

37. Ferrarini, H.; Dissertação de Mestrado, Universidade Federal do Paraná, Brasil, 2004.

38. Morgano, M. A.; Faria, C. G.; Ferrão, M. F.; Bragagnolo, N.; Ferreira, M. M. C.; Ciênc. Tecnol. Aliment. 2005, 25, 25. 


\section{CARACTERIZAÇÃO FÍSICO-QUÍMICA DE QUEIJO PRATO POR ESPECTROSCOPIA NO INFRAVERMELHO E REGRESSÃO DE MÍNIMOS QUADRADOS PARCIAIS}

\section{Elenise Sauer-Leal e Fernanda Martins Okada}

Departamento de Alimentos, Universidade Tecnológica Federal do Paraná, Campus Ponta Grossa, Av. Monteiro Lobato, s/n - km 04, 84016-210 Ponta Grossa - PR, Brasil

Patricio Peralta-Zamora*

Departamento de Química, Universidade Federal do Paraná, CP 19081, 81531-990 Curitiba - PR, Brasil
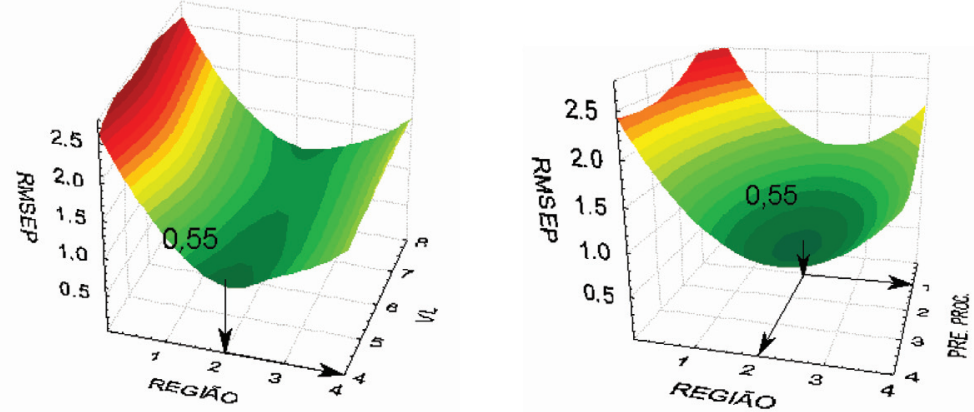

\section{A (Gordura)}
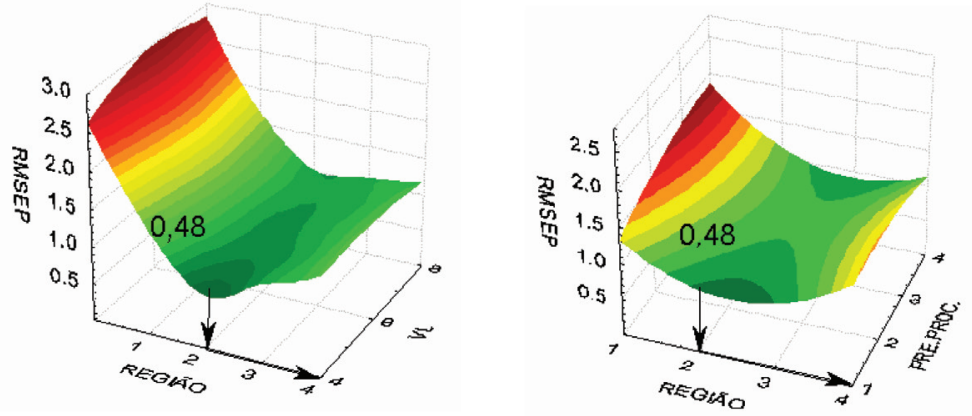

B (Proteínas)
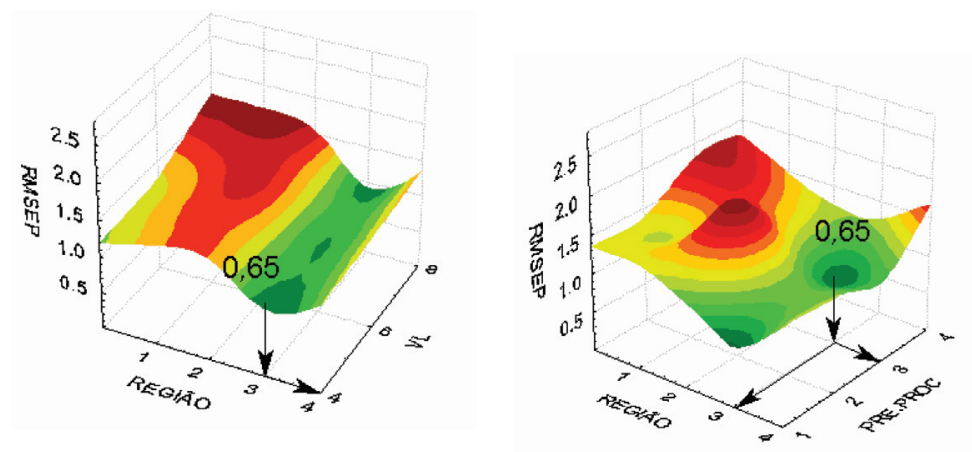

\section{C (Umidade)}

Figura 1S. Efeito do número de variáveis latentes, da região espectral processada e dos tipos de pré-processamento de sinais na capacidade preditiva dos modelos multivariados orientados à determinação do teor de gordura (A), proteínas (B) e umidade (C). Região 1: 1520 a $3760 \mathrm{~cm}^{-1} ; \mathrm{região}^{2:} 4000 \mathrm{a} 6800 \mathrm{~cm}^{-1}$; região 3: 4000 a $7500 \mathrm{~cm}^{-1}$; região 4: 1520 a 3760 e 4000 a $7500 \mathrm{~cm}^{-1}$. Pré-processamento 1: centrado na média 2: alisado e derivado; pré-processamento 3: MSC; pré-processamento 4: MSC e alisamento 

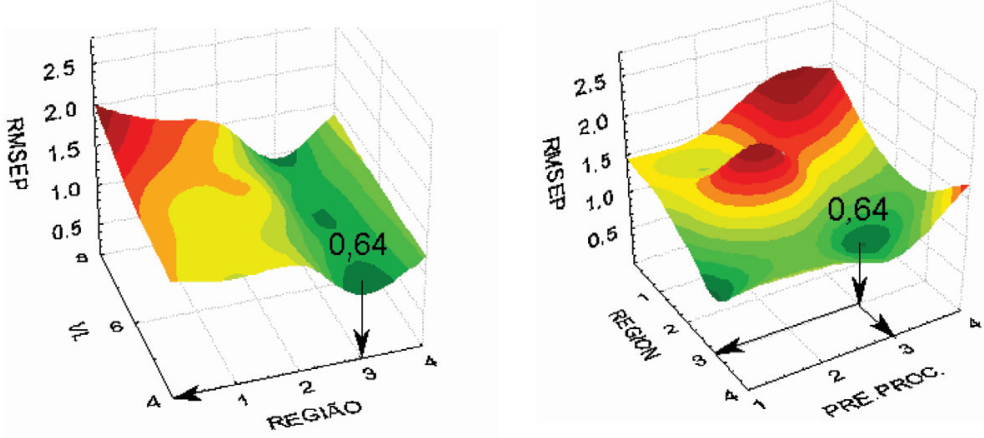

\section{A (Extrato Seco)}
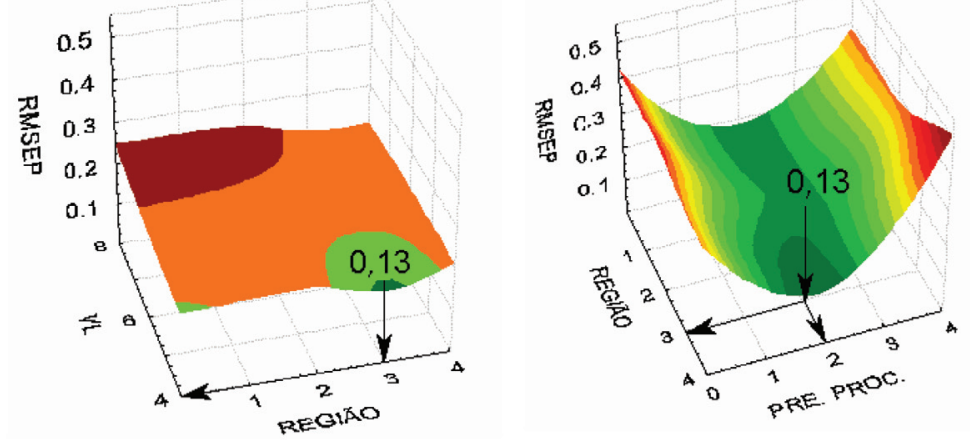

B (Cinzas)
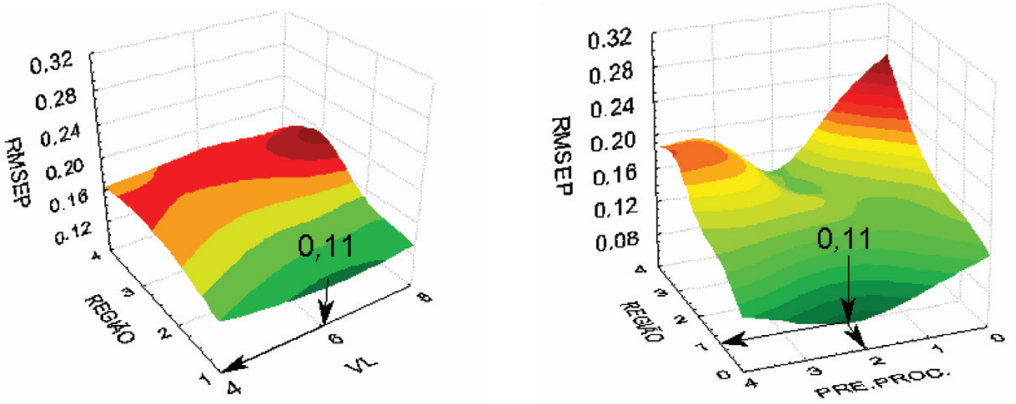

\section{$C(\mathrm{pH})$}

Figura 2S. Efeito do número de variáveis latentes, da região espectral processada e dos tipos de pré-processamento de sinais na capacidade preditiva dos modelos multivariados orientados à determinação do teor de extrato seco (A), cinzas (B) e pH (C). Região 1: 1520 a $3760 \mathrm{~cm}^{-1}$; região $2: 4000$ a $6800 \mathrm{~cm}^{-1}$; região 3: 4000 a $7500 \mathrm{~cm}^{-1}$; região 4: 1520 a 3760 e 4000 a $7500 \mathrm{~cm}^{-1}$. Pré-processamento 1: centrado na média 2: alisado e derivado; pré-processamento 3 : MSC; pré-processamento 4: MSC e alisamento 